\title{
Inversion of the Rectifying Effect in Diblock Molecular Diodes by Protonation
}

\author{
Gustavo M. Morales, Ping Jiang, Shenwen Yuan, Youngu Lee, Arturo Sanchez and Luping Yu*
}

Department of Chemistry and The James Frank Institute, The University of Chicago, 5735 South Ellis

Avenue, Chicago, Illinois 60637.

E-mail: lupingyu@ @idway.uchicago.edu

\section{General.}

Unless otherwise specified, all chemicals were purchased from commercial suppliers and used without further purification. All reactions were conducted under a nitrogen atmosphere. Tetrahydrofuran (THF) was distilled over sodium/benzophenone ketyl under a nitrogen atmosphere

prior to use. ${ }^{1} \mathrm{H}$ NMR spectra were recorded at 400 or $500 \mathrm{MHz}\left({ }^{13} \mathrm{C}\right.$ spectra at 100 or $\left.125 \mathrm{MHz}\right)$ on Bruker DRX-400 or DRX-500 spectrometers, respectively.

\section{Synthesis of the diblock cooligomer diode.}

Based on the sequential deprotection and immobilization procedure we preciously developed, ${ }^{1,2}$ it is necessary to prepare the diode molecules with two different thiol-protected end groups. Compound $\mathbf{9}$ is the target molecule. Our synthetic approach is to make the two blocks with two asymmetric thio protecting groups first, then connect them together through a Stille coupling reaction.

Scheme 1 showed the synthesis of compound 9 . We started with the known compound 4'-methylthio4-bromobiphenyl (1). After the methyl group was removed based on a known procedure, ${ }^{3}$ 4'-bromo-4mercaptobiphenyl (2) was obtained. After deprotonation by sodium hydride and subsequent substitution with cyanoehtylene group, 4-bromo-4'-cyanoethylenethiobiphenyl (3) was prepared that bears the cyanoehtylenethio(CNE) protection group. The next step is transformation to tin compound 4 for Stille coupling reaction. The head-to-head bipyrimidine unit was prepared from a coupling reaction: first, 2-chloro-5-bromopyrimidine $\mathbf{5}$ was treated with tributyltin lithium at low temperature, leading to selective substitution at the 5 position; then react with 2-iodo-5-bromo pyrimidine, with its 2-iodo position more reactive than the 5-bromo position in the stille coupling conditions, therefore the head-tohead bipyrimidine 7 that still have two end functional groups was prepared. Trimethylsilylethylenethio 
protecting group was then added through a previously developed procedure to the more reactive 2chloro positions, thus compound $\mathbf{8}$ was obtained almost quantitatively. ${ }^{4}$ The final step is to couple these two components $\mathbf{4}$ and $\mathbf{8}$ together by a Stille reaction to get the target compound $\mathbf{9}$.

Scheme 1.

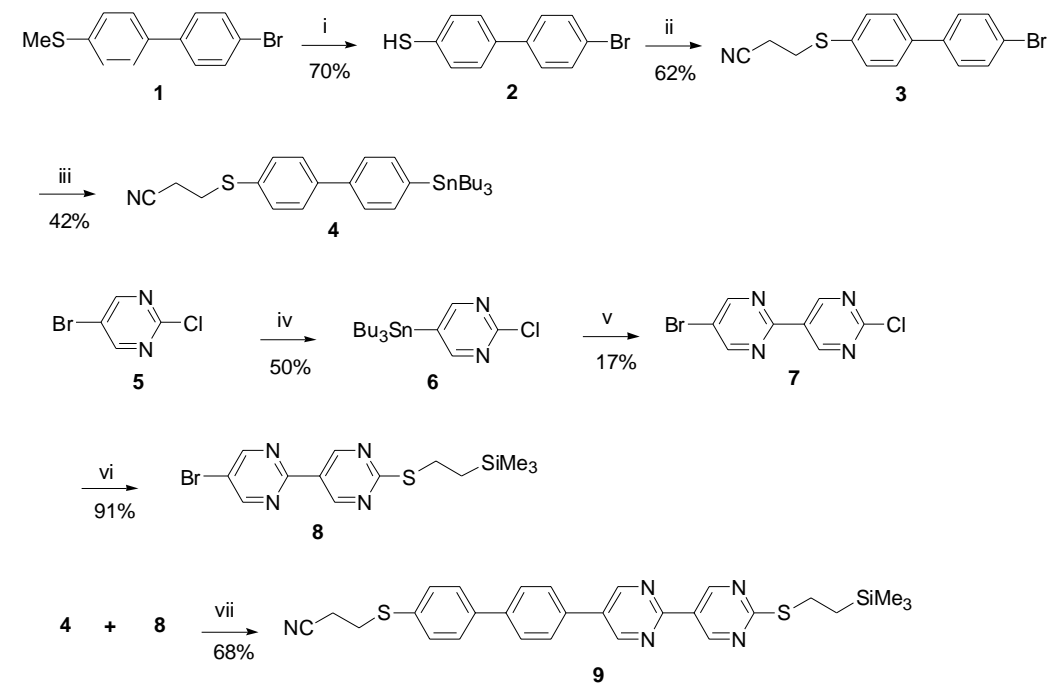

i. $\mathrm{mCPBA} / \mathrm{CH}_{2} \mathrm{Cl}_{2},\left(\mathrm{CF}_{3} \mathrm{CO}\right)_{2} / \mathrm{CH}_{2} \mathrm{Cl}_{2}, \mathrm{MeOH} / \mathrm{TEA}$. ii. 1). $\mathrm{NaH}, \mathrm{DMF}$, 2). $\mathrm{BrCH}_{2} \mathrm{CH}_{2} \mathrm{CN}$. iii. $(\mathrm{Bu} 3 \mathrm{Sn})_{2}, \mathrm{Pd}\left(\mathrm{PPH}_{3}\right)_{4}$, toluene. iv. $\mathrm{Bu}_{3} \mathrm{SnLi},-78^{\circ} \mathrm{C}$. v. $\mathrm{PdCl}_{2}\left(\mathrm{PPh}_{3}\right)_{2}$, 2-iodo - 5-bromopyrimidine. vi. $\mathrm{HSCH}_{2} \mathrm{CH}_{2} \mathrm{SiMe}_{3}$, Cul, Neocuproine, $\mathrm{NaO}^{\mathrm{t}} \mathrm{Bu}$, Toluene. vii. $\mathrm{Pd}\left(\mathrm{PPH}_{3}\right)_{4}$, Toluene.

Synthesis of 4'-Bromo-biphenyl-4-thiol (Compound 2): 4'-methylthio-4-bromobiphenyl (1) ${ }^{5}$ $(1.07 \mathrm{~g}, 3.85 \mathrm{mmole})$ in dichloromethane $(50 \mathrm{ml})$ under ice-water bath was added with mCPBA $(1.0 \mathrm{~g}, 5.8$ mmole). After stirring 30 minutes, $\mathrm{Ca}(\mathrm{OH})_{2}(481 \mathrm{mg}, 6.5 \mathrm{mmole})$ was added. After filtration, to the filtrate was added trifluoroacetoanhydride $(1.13 \mathrm{ml}, 8.0 \mathrm{mmole})$ and the reaction mixture was refluxed for 1.5h. After the solvents were evaporated, the residue was redissolved in the mixed solution (50:50, v/v) of methanol and triethylamine (50ml). Pure compound 2 (710 mg, 70\% yield) was obtained from column chromatography (silical gel, hexane/ethyl acetate $=10 / 1) .{ }^{1} \mathrm{H} \mathrm{NMR}\left(\mathrm{CDCl}_{3}, \mathrm{ppm}\right)^{5}: 3.50(\mathrm{~s} 1 \mathrm{H})$, 7.41(AB, J = 8.6Hz, v = 64Hz, 4H), 7.43(m, 4H).

Synthesis of 3-(4'-Bromo-biphenyl-4-ylsulfanyl)-propionitrile (Compound 3): compound 2 (710mg, 2.69mmole) in anhydrous DMF (15ml) was added sodium hydride (72mg, 3.0mmole). After the reaction mixture was stirred at room temperature for about an hour, 3-bromopropionitrile (3.3mmole) was added to the reaction solution. After workup and column chromatography (silica gel, hexane/ethyl acetate $=4 / 1)$, pure compound $3\left(530 \mathrm{mg}, 62 \%\right.$ yield) was obtained as white solid. ${ }^{1} \mathrm{H}$ NMR $\left(\mathrm{CDCl}_{3}, \mathrm{ppm}\right): 2.62(\mathrm{t}, \mathrm{J}=7.3 \mathrm{~Hz}, 2 \mathrm{H}), 3.15(\mathrm{t}, \mathrm{J}=7.3 \mathrm{~Hz}, 2 \mathrm{H}), 7.42$ (m, 8H, Ph). MS (EI): Calcd, 316.99; found, 317.0.

Synthesis of 3-(4'-Tributylstannanyl-biphenyl-4-ylsulfanyl)-propionitrile (Compound 4): The solution of compound 3 (530 mg, $1.6 \mathrm{mmol}), \mathrm{Pd}\left(\mathrm{PPh}_{3}\right)_{4}(93 \mathrm{mg}, 0.08 \mathrm{mmole})$ and bis(tributyltin)(1.05g, 
$1.8 \mathrm{mmole})$ in dry toluene $(15 \mathrm{ml})$ was refluxed at $120{ }^{\circ} \mathrm{C}$ for overnight. The reaction mixture was then cooled down to room temperature and filtered out. The filtrate was concentrated in vacuum and purified by column chromatography (silica gel, hexane/ethyl acetate $=4 / 1)$. Thus pure compound 4(354 mg, $42 \%)$ was obtained. ${ }^{1} \mathrm{H}$ NMR $\left(\mathrm{CDCl}_{3}, \mathrm{ppm}\right)$ : 0.83-0.94 (m, 9H), 1.06-1.09(m, 6H), 1.31-1.38(m, 6H), 1.54-1.57(m, 6H), 2.62(t, J=7.3Hz, 2H), 3.15(t, J=7.3Hz, 2H), 7.46-7.60 (m, 8H, Ph).

Synthesis of compound 2-chloro-5-tributylstananylpyrimidine (Compound 6): ${ }^{6}$ A solution of 2chloro-5-bromopyrimidine (5) (4.5mmole) in THF (10ml) was added dropwise, with vigorous stirring, to a solution of tributylstannyllithium $(5 \mathrm{mmole})$ in $\mathrm{THF}(10 \mathrm{ml})$ maintained at $-78{ }^{\circ} \mathrm{C}$ under nitrogen. The reaction was monitored by TLC. When no further changes were observed, the reaction mixture was allowed to warm up to r.t. and quenched with $\mathrm{NH}_{4} \mathrm{Cl}$ aqueous solution and extracted with ethyl acetate, washed with water and dried over sodium sulfate. 2-chloro-5-tributylstannylpyrimidine 6 was obtained from column chromatography ( silica gel, hexane/ethyl acetate $=4 / 1$ ) in 50\% yield. ${ }^{1} \mathrm{H} \mathrm{NMR}\left(\mathrm{CDCl}_{3}\right.$, ppm): 0.88-0.94(m, 9H), 1.13-1.16(m, 6H), 1.30-1.40(m, 6H), 1.47-1.51(m, 6H), 8.56(s, 2H).

Synthesis of 5-Bromo-2'-chloro-[2,5']bipyrimidinyl (Compound 7): 2-iodo-5-bromopyrimidine ${ }^{7}$ (752mg, 2.64mmole), compound 6(900mg, 2.2 mmole ), $\mathrm{Pd}\left(\mathrm{PPh}_{3}\right)_{2} \mathrm{Cl}_{2}(90 \mathrm{mg}, 5 \%)$ and $\mathrm{PPh}_{3}(34 \mathrm{mg}$, $10 \%)$ in dry toluene $(20 \mathrm{ml})$ was refluxed at $120{ }^{\circ} \mathrm{C}$ for overnight. The reaction mixture was then cooled down to room temperature and filtered out. The filtrate was concentrated in vacuum and purified, followed by column chromatography ( silica gel, hexane /ethyl acetate $=4 / 1)$. Pure compound 7(91 mg, $17 \%)$ was obtained as an yellowish solid. ${ }^{1} \mathrm{H} \mathrm{NMR}\left(\mathrm{CDCl}_{3}, \mathrm{ppm}\right): 8.90(\mathrm{~s}, 2 \mathrm{H}), 9.55(\mathrm{~s}, 2 \mathrm{H}) .{ }^{13} \mathrm{C} \mathrm{NMR}$ $\left(\mathrm{CDCl}_{3}, \mathrm{ppm}\right): 120.2,128.7,158.2,158.4,159.2$, 163.1.MS: Calcd, 269.9. Found: $(\mathrm{M}+1)^{+}, 271.0$.

\section{Synthesis of compound 5-Bromo-2'- (2-trimethylsilanyl-ethylsulfanyl)-[2,5'] bipyrimidinyl} (Compound 8): Under nitrogen sodium tert-butoxide (48 mg, 1.5eq.), CuI (6.3mg, 10\%) and neocuproine $(7.2 \mathrm{mg}, 10 \%)$ were added into a $100 \mathrm{ml}$ flame-dried Schlenk flask equipped with Teflon stir bar. Compound 7 (81mg, $0.3 \mathrm{mmole})$ and 2-trimethylsilyl-ethanethiol (40 $\mathrm{mg}, 0.3 \mathrm{mmole})$ in anhydrous toluene $(10 \mathrm{ml})$ were injected into the flask through a septum. The contents were then stirred at $110{ }^{\circ} \mathrm{C}$ for 24 hours. The reaction mixture was then cooled to room temperature and filtered to remove any insoluble residues. The filtrate was concentrated in vacuo and was purified by column chromatography (silica gel, hexane/ethyl acetate =4/1). Pure compound 8 (100mg, 91\%) was obtained.

${ }^{1} \mathrm{H}$ NMR $\left(\mathrm{CDCl}_{3}, \mathrm{ppm}\right):$ 0.08(s, 9H), 1.04-1.07(m, 2H), 3.23-3.27(m, 2H), 8.79(s, 2H), 9.42(s, 2H). ${ }^{13} \mathrm{C}$ NMR $\left(\mathrm{CDCl}_{3}, \mathrm{ppm}\right):-1.8,16.6,27.4,118.9,124.8,156.6,158.1,159.4,175.3$. MS: Calcd, 368.0. Found, $(\mathrm{M}+1)^{+}, 368.9$

\section{Synthesis of 3-\{4'-[2'-(2-Trimethylsilanyl-ethylsulfanyl)-[2,5']bipyrimidinyl-5-yl]-biphenyl-4-} ylsulfanyl\}-propionitrile (Compound 9): A mixture of compound 4(148 mg, 0.28mmole), compound 8 (100mg, 0.272mmole) and $\mathrm{Pd}\left(\mathrm{PPh}_{3}\right)_{4}(16 \mathrm{mg})$ were dissolved in anhydrous toluene $(10 \mathrm{ml})$. The 
resulting mixture was heated at $120{ }^{\circ} \mathrm{C}$ for 12 hours, and then was cooled to room temperature. Toluene was removed under high vacuum and chloroform was added to the residue, and resulting mixture was filtered through a pad of silica gel. The crude product was purified by column chromatography (silica gel, chloroform/ethyl acetate $=3 / 1)$ to afford pure compound $9(96 \mathrm{mg}, 68 \%) . \quad{ }^{1} \mathrm{H} \mathrm{NMR}\left(\mathrm{CDCl}_{3}\right.$, ppm): 0.10(s, 9H), 1.05-1.10(m, 2H), 2.66(t, J=7.2Hz, 2H), 3.20(t, J=7.2Hz, 2H), 3.25-3.30(m, 2H), 7.52(d, J=8.3Hz, 2H, Ph), 7.62(d, J=8.3Hz, 2H, Ph), 7.71-7.77(m, 4H, Ph), 9.06(s, 2H, Py), 9.52(s, 2H, Py). ${ }^{13} \mathrm{C} \mathrm{NMR}\left(\mathrm{CDCl}_{3}, \mathrm{ppm}\right):-1.7,16.7,18.3,27.4,30.1,117.9,125.6,127.2,127.9,128.0,131.6$, 131.8, 133.0, 133.3, 139.3, 140.7, 155.1, 156.7, 160.1, 174.9. MS: Calcd, 527.2. Found, $(\mathrm{M}+1)^{+}$, 527.9 .

Substrate Preparation: The gold electrodes used in the STM studies were grown by gold (99,999\%) deposition from a tungsten boat onto freshly cleaved mica (highest quality V1, Ted Pella, Inc.). Before deposition, the mica was heated at a temperature of $\sim 450^{\circ} \mathrm{C}$ overnight under high vacuum. ${ }^{8}$ Then, gold film was annealed in the same atmosphere at $\sim 400^{\circ} \mathrm{C}$ for $10 \mathrm{~h}$. and stored under Argon. With exposure to atmospheric air, the surface becomes contaminated by adoption of carbonaceous materials, ${ }^{9}$ which are removed by subjecting the gold surface to hydrogen flame annealing until the film radiates a dim orange color (in a dark room). Then, the film is quenched in pure methanol saturated with Argon. The substrates used in the grazing incidence Fourier transform infrared spectroscopy (GI-FTIR) studies were prepared by evaporation of gold $(99,999 \%)$ onto chromium coated silicon wafers ( $\mathrm{Si} / \mathrm{Cr} / \mathrm{Au})$ using a method similar to the previously detailed in the literature. ${ }^{10}$ Prior to their use the gold substrates were cleaned in a mixture of $\mathrm{H}_{2} \mathrm{SO}_{4}-\mathrm{H}_{2} \mathrm{O}_{2}$ (7:1) (CAUTION: After use this solution, it must not be stored as explosive oxo-peroxides may form) at $90^{\circ} \mathrm{C}$ for 5 minutes. Then, the substrates were rinsed with ultrapure water and dried in $\mathrm{N}_{2}$.

Monolayer Preparation: The self-assembled process was carried out at room temperature under $\mathrm{N}_{2}$ atmosphere. Immediately after the annealing process, the gold substrates were transferred from ethanol to a solution of DDT/molecule 9 in a concentration ratio of 1:500 (5 ml of DDT $\sim 10^{-3} \mathrm{M}$ in ethanol and $5 \mathrm{ml}$ of diodes molecule $\sim 5 \times 10^{-6} \mathrm{M}$ in THF with an excess of a fresh prepared sodium ethoxide in ethanol). After 14-18 $\mathrm{h}$ the electrode was removed from the solution, rinsed five times with pure ethanol, hexane, ethanol again and finally fresh distilled THF. This procedure removes the protection group cyanoethylene in the molecules leaving the group trimethylsilylethylene intact, and guarantees the correct orientation of the molecules on the metallic surface. The trimethylsilylethylene protecting group can be cleaved now by dipping the electrode with the codeposited SAM/Au into a $5 \mathrm{mM}$ solution of TBAF in THF for 6-7 $\mathrm{h}$ and afterwards rinsed several times with pure THF saturated with argon. The exposed $\mathrm{S}^{-}$anion or $\mathrm{SH}$ groups can be covalently bonded to the gold nanoparticles, the electrode is immersed in a fresh prepared solution of gold nanoparticles in toluene ${ }^{11}$ (concentration corresponding 
to an absorbance 0.1 at $520 \mathrm{~nm}$ ) for 1-2 $\mathrm{h}$. and subsequently rinsed with tetraoctylammonium bromide in toluene (several times), then pure toluene, THF and methanol. Finally, the sample is submerged in a solution of $\mathrm{NaOEt}$ in ethanol for $6 \mathrm{~h}$, subsequently washed with abundant pure ethanol then dried with a continuous and gentle flow of argon and immediately used for STM and STS measurements.

Protonation of diode molecules: The samples were submerged in a solution of $\mathrm{HClO}_{4} 0.1 \mathrm{M}$ in ethanol for 4-6 h, after which they were washed with pure methanol and dried.

Deprotonation of diode molecules: The protonated samples were treated with a solution of NaOEt in ethanol for $6 \mathrm{~h}$, washed with pure ethanol and dried.

Grazing Incidence FT-IR: The GI-FTIR data were recorded under a nitrogen atmosphere in a Nicolet Magna Spectrometer equipped with a FT-85 fixed $85^{\circ}$ grazing angle accessory (Thermo Spectra-Tech). Prior to the measurement of the SAMs on gold-coated silicon wafers, a reference spectrum was recorded on a freshly cleaned gold substrate. Before each acquisition, the sample chamber was purged with dry $\mathrm{N}_{2}$ until a constant spectrum was obtained during 100 consecutive scans (1-2 h.). Five thousands consecutive scans were averaged for the sample and reference spectra, the spectra resolution was set at $4 \mathrm{~cm}^{-1}$.

Figure 1 displays the infrared spectra of a diode molecules SAM before and after the TMSE deprotection by TBAF, for comparison, the transmission spectra of pure diode molecules on $\mathrm{KBr}$ is also displayed. The arrow shown the absorption band from the $-\mathrm{Si}-\mathrm{CH}_{3}$ moiety assigned to the $\mathrm{C}-\mathrm{H}$ symmetric deformation vibration, which was considerably reduced after the TMSE cleavage. On the top, Figure 1 shows the FTIR spectrum of the TMSE deprotected monolayer after protonation by perchloric acid in ethanol. The spectrum clearly shows the appearance of an absorption band assigned to the $\mathrm{ClO}_{4}{ }^{-}$anion, witch disappear after deprotonation with $\mathrm{NaOEt}$ in ethanol. 


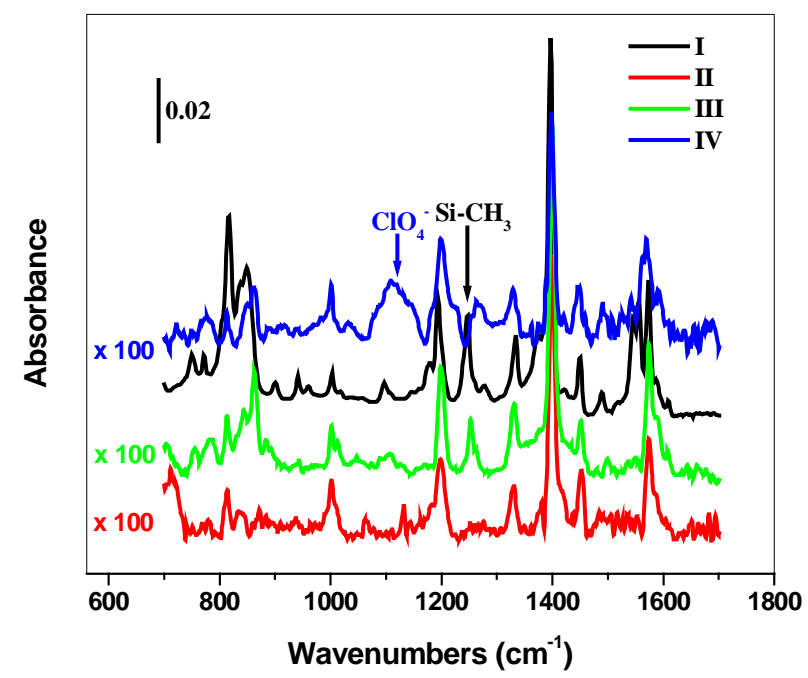

Figure 1. Infrared spectra of the $700-1700 \mathrm{~cm}^{-1}$ region of (I) compound 3 in $\mathrm{KBr}$, a SAM of compound 9 on $\mathrm{Au}$ (II) after and (III) before TMSE cleavage. (IV) After protonation by $\mathrm{ClO}_{4} \mathrm{H}$.

UV-visible spectroscopy: The absorption spectra were recorded using a Shimadzu UV-2401PC UV-visible spectrophotometer. Figure 2 shows the UV-visible spectra of diode molecules in THF solution. The neutral state exhibited a band with absorption maximum at $332 \mathrm{~nm}$ (Spectrum I) witch has been assigned to a $\pi \rightarrow \pi^{*}$ transition. ${ }^{12}$ After addition of an excess of perchloric acid this band was red shifted and the absorbance decreased reaching an absorption maximum at $340 \mathrm{~nm}$ (Spectrum II). Titration with $\mathrm{NaOEt}$ returned the band reversibly to the original position (Spectrum III-VI).

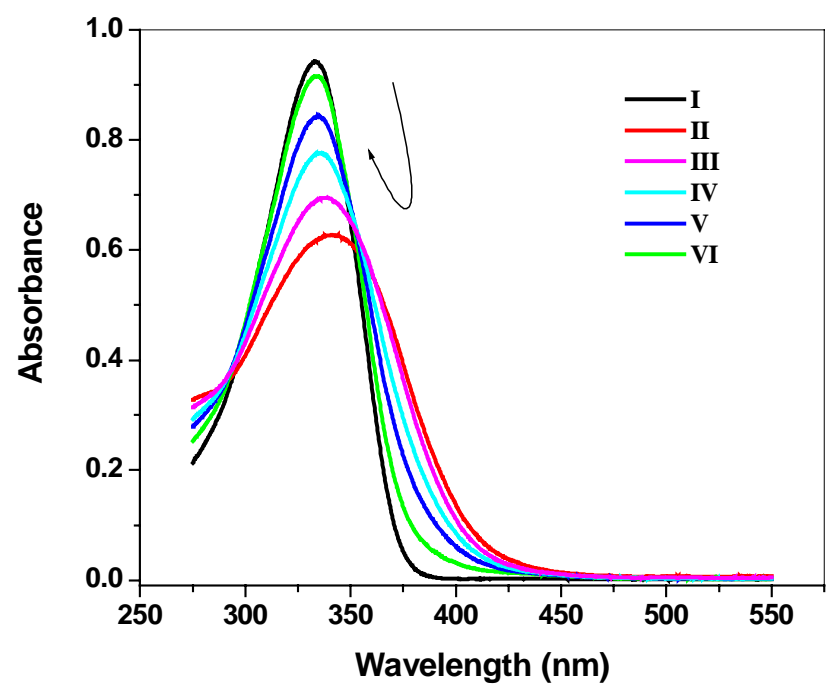

Figure 2. UV-visible spectra of diode molecules in THF before (I) and after (II) $\mathrm{ClO}_{4} \mathrm{H}$ addition. Titration of the protonated solution with NaOEt. (III $\rightarrow$ IV). 
Scanning Tunneling Microscopy (STM): STM measurements were carried out by utilizing a NanoScope III STM (Digital Instrument) equipped with a low current STM head and Picoamp Boos Box (Digital Instrument), which allows STM measurements with tunneling currents in the pA range. The tips used in the measurements were fabricated by electrochemical etching of a $\mathrm{Pt} / \mathrm{Ir}$ wire (Molecular Imaging, Phoenix, AZ, USA) in $8 \mathrm{M} \mathrm{NaOH}$. After etching, the tips were rinsed with deionized water and immediately mounted in the STM converter head. The experiments presented here were performed in atmospheric air at room temperature and the images were recorded in constant current operating mode.

Scanning tunneling spectroscopy (STS): STS data were acquired using a NanoScope software version 5.12 (Digital Instrument) working in the I(V) mode. This operation mode allows monitoring the variation of the tunneling current (I), due to variations of the bias voltage (V). After the tip was positioned at a specific point on the surface, the feedback is shut off and a spectroscopic plot is acquired (the tunnel current is measured as the sample voltage is ramped at $10 \mathrm{Vs}^{-1}$ ). In all the experiments, the bias potential was applied to the sample with respect to the grounded tip. In this configuration, a positive bias corresponds to an electron flow from the tip to the sample and in the opposite way for negative bias voltage. The STS data showed are averaged I(V) curves, measured positioning the STM tip on more than fifteen immobilized individual AuNPs in three samples independently prepared. The I(V) data for each Au NP is the average of about 100 individual I(V) curves, each of which is composed of 256 points, while each point has been averaged 3-5 times by the software.

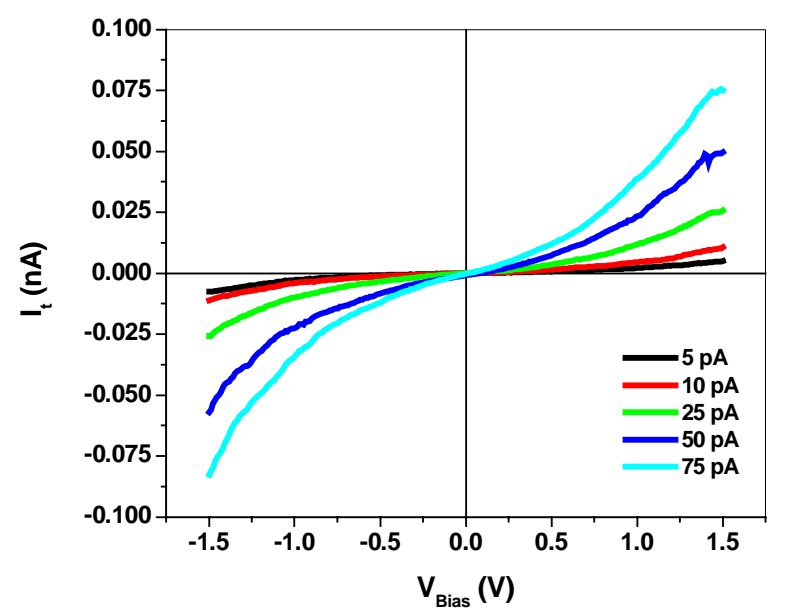

Figure 3. Characteristic $\mathrm{I}(\mathrm{V})$ data taken at different current set points for a $\mathrm{V}_{\mathrm{Bias}}$ of $1.5 \mathrm{~V}$ on the dodecanethiol on the same sample used for the assemblies.

In order to avoid artifacts, the tips used for the STS characterizations were previously examined according to the following procedure: In the alkanethiol region, the tip must be able to produce 
atomically resolved images with the characteristic $(3 \times 3) \mathrm{R} 30^{\circ}$ layer structure of alkanethiolate on $\mathrm{Au}$ (111). Finally, before and after the STS measurement on the nanoparticle the I(V) curve on the DDT region, using the same tip, should be almost symmetric according to the previously reported behavior in these systems (Figure 3). ${ }^{13}$ The behavior of the junction under different gap conditions was also investigated. Figure 4 shows the I(V) curves obtained at different current set points (distance between the STM tip and the Au NP) for the protonated and unprotonated assembly. The opposite asymmetric I(V) behaviors for both assemblies were observed consistently at various current set points.
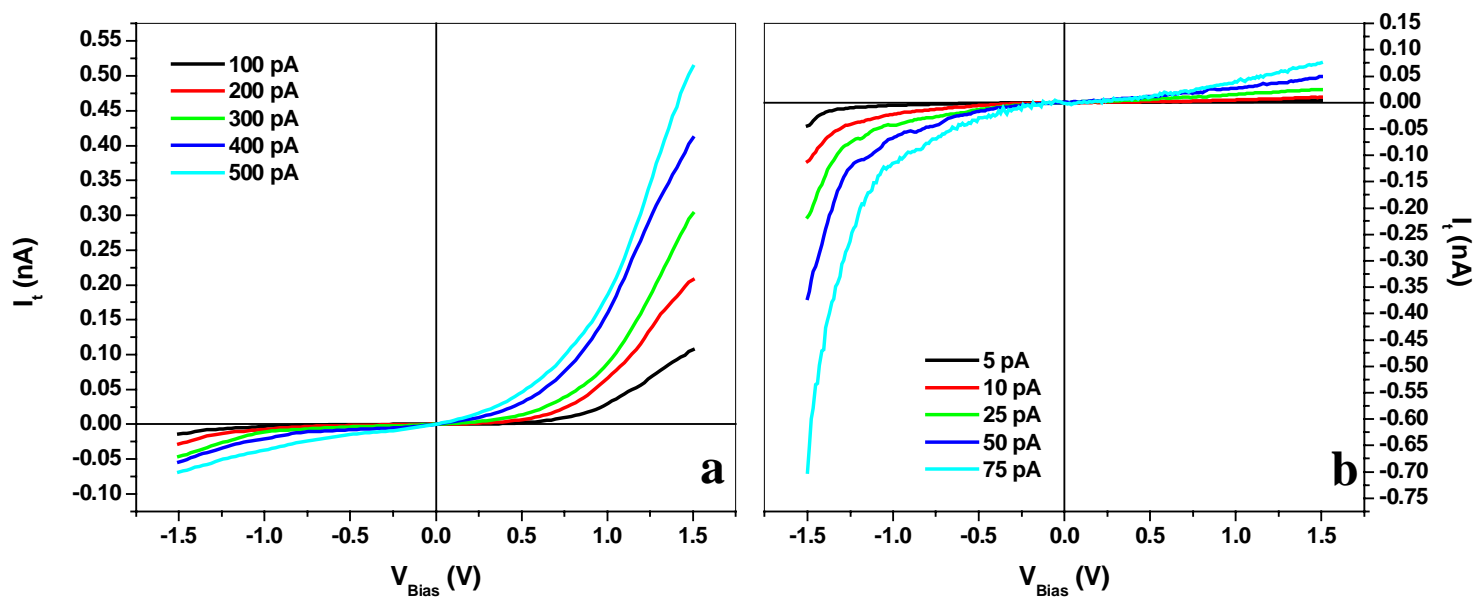

Figure 4. $\mathrm{I}(\mathrm{V})$ data taken at different current set point for a $\mathrm{V}_{\text {Bias }}$ of $1.5 \mathrm{~V}$ for the assembly (a) before and (b) after protonation by $\mathrm{ClO}_{4} \mathrm{H}$.

Quantum calculations: The molecular properties of diblock molecules were calculated using the B3PW91 hybrid DFT functional with the basis set LANL2DZ as implemented in the Gaussian 98 modeling package. Two sets of calculations were performed for each diblock molecule. The first set involved computing molecular properties without any gold clusters attached to the molecule. In the second set, a cluster of three gold atoms, representing a $\langle 1,1,1\rangle$ surface was attached to both sulfur atoms at the end of the molecule. Data from the first set was used to correct for the appearance of metal induced gaps states caused by addition of gold, to give the true molecular electronic structure of the molecule in the second set of calculations.

1 S. K.; Pollack, Naciri, J.; Mastrangelo, J.; Patterson, C. H.; Torres, J.; Moore, M.; Shashidhar, R.; Kushmerick, J. G.; Langmuir; 2004; $20(5) ; 1838-1842$.

2 Jiang, P.; Morales, G. M.; You, W.; Yu, L. Angew. Chem., Int. Ed. Engl. 2004, 43, 4471.

3 Yu, C. J.; Chong, Y.; Kayyem, J. F.; Gozin, M. J. Org. Chem., 1999, 64 (6), 2070.

4 Bates, C. G.; Gujadhur, R. K.; Venkataraman, D. Org. Lett., 2002, 4 (16), 2803.

5 Kang, J. F.; Ulman, A.; Liao, S.; Jordan, R.; Yang, G.; Liu, G.-y. Langmuir, 2001, 17 (1), 95. 
6 Sandosham, J.; Undheim, K. Tetrahedron, 1994, 50 (1), 275.

7 Goodby, J. W.; Hird, M.; Lewis, R. A.; Toyne, K. J. Chem. Commun., 1996, 2719.

8 DeRose, J. A.; Thundat, T.; Nagahara, L. A.; Linsay, S. M. Surf. Sci. 1991, 256, 102.

9 Kolb, D. M.; Dakkouri, A. S.; Batina, N. In: Nanoscale Probes of the Solid/Liquid Interface, NATO ASI Series E288, A. A. Gerwirth H. Siegenthaler, Eds.; Kluwer, Dordrecht, 1995.

10 Zehner, R. W.; Sita, L. R. Langmuir; 1997, 13(11), 2973

11 Gittins, D.I.; Caruso F. Angew. Chem. Int. Ed. 2001, 40, 3001.

12 Vaschetto, M. E.; Springborg, M.; Monkman, A. P. J. Mol. Struct. (Theo. Chem.) 1999, 468, 181.

13 Cui, X. D.; Primak, A.; Zarate, X.; Tomfohr, J.; Sankey, O. F.; Moore, A. L.; Moore, T. A.; Gust, D.; Harris, G.; Lindsay, S. M.; Science, 2001, 294, 571. 